\title{
Quel changement à l'ère numérique? Quelle ingénierie pédagogique pour y répondre?
}

\author{
What change in the the digital age? What instructional \\ engineering to answer this question?
}

\section{¿Qué cambio en la era digital? ¿Qué ingeniería pedagógica para responder a ello?}

RÉSUMÉ

À l'ère numérique, apprendre exige un solide savoir-faire informationnel et la maitrise d'opérations cognitives pour repérer, explorer, départager, choisir, lier, croiser, analyser et traiter le foisonnement des contenus. Dans ce contexte éclaté et transformé, les contenus et les processus d'apprentissage ne sont plus les mêmes. L'idée de l'apprenant autonome, très souvent mise de l'avant depuis bon nombre d'années, prend désormais place, impose tout son sens et invite à redéfinir le paradigme de formation. De manière plus concrète, il est question de s'engager dans une réingénierie de la formation qui soit authentiquement ouverte à l'usage du numérique; une réingénierie dont la profondeur et la complexité ne sont pas à sous-estimer; une réingénierie qui remet en cause tant la finalité que les moyens de formation.

Mots-clés: Ingénierie pédagogique, méthode d'ingénierie pédagogique, paradigme de formation, apprentissage autonome, apprentissage à l'ère numérique 
In the digital age, learning requires strong informational skills and mastery of cognitive operations to identify, explore, separate, choose, link, cross-reference, analyze and process the proliferation of content. In this fragmented and transformed context, the contents and learning processes are no longer the same. The idea of the autonomous learner, very often put forward for many years, is now taking place, imposing its full meaning and inviting us to redefine the training paradigm. More concretely, it is a question of engaging in a reengineering of training that is authentically open to the use of digital technology; a reengineering whose depth and complexity should not be underestimated; a reengineering that calls into question both the purpose and the means of training.

Keywords: instructional engineering, instructional engineering method, training paradigm, autonomous learning, learning in the digital age

\section{RESUMEN}

En la era digital, el aprendizaje requiere grandes habilidades informativas y dominio de las operaciones cognitivas para identificar, explorar, separar, elegir, vincular, cruzar referencias, analizar y procesar la proliferación de contenidos. En este contexto fragmentado y transformado, los contenidos y los procesos de aprendizaje ya no son los mismos. La idea del aprendiz autónomo, planteada muy a menudo desde hace muchos años, se está llevando a cabo, imponiendo todo su significado e invitándonos a redefinir el paradigma de la formación. Más concretamente, se trata de una reingeniería de la formación auténticamente abierta al uso de la tecnología digital; una reingeniería cuya profundidad y complejidad no debe subestimarse; una reingeniería que cuestiona tanto el propósito como los medios de la formación.

Palabras clave: Ingeniería pedagógica, método de ingeniería pedagógica, paradigma de formación, aprendizaje autónomo, aprendizaje en la era digital

\section{Introduction : apprendre dans une ère d'abondance de contenus}

À l'ère numérique, les contenus sont désormais d'une telle abondance qu'on réfère aux ordinateurs quantiques pour pouvoir les analyser (Carricano \& de Lassence, 2009). Ils sont disponibles sur une variété de supports et sont de natures diverses ; il peut s'agir de données, d'informations, de connaissances ou de savoirs. Apprendre dans ce contexte exige un solide savoir-faire informationnel et la maitrise d'opérations cognitives pour repérer, explorer, départager, choisir, lier, croiser, analyser et traiter le foisonnement des contenus. II en résulte une construction personnelle, plus ou moins utile selon les compétences de l'apprenant et l'orientation qu'il souhaite donner à son apprentissage.

Apprendre à l'ère numérique ne pose pas qu'un défi à l'apprenant. Ce processus inédit impacte la tâche de l'enseignant et la rend plus complexe. Comme le souligne Cristol (2019), l'enseignant conserve son rôle de médiateur, mais il requiert désormais de sa part un effort pour cartographier et se diriger sur le 
volcan informationnel. La construction même des contenus est bouleversée faisant appel à un processus d'apprentissage inédit. L'apprenant doit pouvoir distinguer les informations utiles, pertinentes ou essentielles de celles qui sont gratuites, trompeuses, erronées ou sans fondement. II doit pouvoir mobiliser des compétences informationnelles et des savoir-faire nouveaux pour interpréter et produire des contenus dans un écosystème informationnel propre à l'ère du numérique.

Par ailleurs, en classe, les stratégies pédagogiques se sont enrichies pour ne plus se limiter à l'approche transmissive et magistrale. Ces stratégies intègrent des interactions élargies et enrichies en soutien aux apprentissages sociaux et participatifs, collectifs et collaboratifs, formels et non-formels, dans des espaces présentiels et virtuels. Selon Cristol (2019), cette pédagogie plus ouverte à laquelle se jouxte un processus d'apprentissage exploitant les ressources du numérique contribue à modifier les rapports enseignants/enseignés. Les connaissances partagées circulent et s'enrichissent hors du contrôle de l'enseignant. Une compétition est dès lors engagée entre la parole d'autorité de l'enseignant et les sources en ligne, perturbant le passage du savoir vers l'apprenant.

Dans ce contexte éclaté et transformé, les contenus et les processus d'apprentissage ne sont plus les mêmes. L'idée de l'apprenant autonome, très souvent mise de l'avant depuis bon nombre d'années, prend désormais place et impose tout son sens. Les changements observés nous interpellent. lls ouvrent la voie à un possible qui reste à préciser, à opérationnaliser et à implanter dans une perspective institutionnelle. De manière plus concrète, il est question de s'engager dans une réingénierie de la formation qui soit authentiquement ouverte à l'usage du numérique, en soutien au développement de l'autonomie et de l'apprentissage autonome non seulement dans le cadre de la formation, mais aussi tout au long de la vie.

II s'agit d'une réingénierie dont la profondeur et la complexité ne sont pas à sous-estimer ; une réingénierie qui remet en cause tant la fin que les moyens de formation. II ne s'agit surtout pas d'instrumenter les systèmes éducatifs avec les nouveaux outils de l'ère numérique tout en conservant le modèle dominant.

\section{Recadrage de la formation et de sa finalité}

II y a presque vingt ans, Monique Linard (2002) soutenait que le développement de l'autonomie de l'apprenant devrait être la préoccupation première ; que l'apprenant est de loin son meilleur pilote et que pour apprendre, il ne faut pas nécessairement « être enseigné ». Dans cette perspective, elle proposait que l'apprenant soit invité à évoluer dans des environnements humains et techniques favorables, compétents et bienveillants. Plus encore, elle précisait que l'apprenant devrait pouvoir apprendre par luimême dans ces environnements auto-organisés. Si cette proposition pouvait apparaître utopique à l'époque, elle s'avère aujourd'hui envisageable. Il est désormais possible de concevoir des environnements numériques qui font place à l'autonomie, gérés par l'apprenant, offrant des ressources pour l'amener à apprendre par lui-même. Toutefois, force est de constater que dans nos systèmes éducatifs l'autonomie de l'apprenant comme principe moteur de la formation ne va pas automatiquement de soi. La proposition pose problème.

\subsection{Problématique de l'autonomie}

M. Linard (2003, p. 4) définit l'autonomie comme une métacompétence, une " capacité de haut niveau, cognitive mais aussi psychologique et sociale, qui implique des qualités d'attention, d'autocontrôle, d'intelligence, de confiance en soi et de relation que peu d'individus possèdent ensemble à l'état naturel ». Elle en traite comme d'un concept réflexif circulaire, de soi sur soi, donc nécessairement complexe. Si l'autonomie s'avère complexe comme phénomène, son application comme mode d'apprentissage est problématique. Comment développer cette capacité? De quelle liberté l'apprenant peut-il se prévaloir? La 
marge de liberté se limite-t-elle aux apprentissages prescrits ou va-t-elle au-delà? La nature et l'étendue de la liberté soulèvent un problème de compatibilité face aux normes actuelles, aux règles qui régissent les systèmes éducatifs allant même jusqu'à remettre en cause l'organisation sociale.

La problématique de l'autonomie et son développement devient encore plus complexe et incontournable selon Linard (2004) lorsqu'elle est croisée avec l'usage des technologies comme outils privilégiés de l'activité autonome.

L'instrumentation massive des activités par les TIC bouleverse en profondeur les conditions de production et de transmission des activités et des connaissances... [elle] oblige à prendre en compte, dans les conceptions théoriques et dans les pratiques sociales, les nouvelles formes d'autorégulation nécessaires aux nouvelles formes de complexité et d'autonomie suscitées par la généralisation des TIC [...] la puissance des moyens [technologiques] débordant tout contrôle, elle se développe pour son propre compte et tend à devenir à elle-même sa propre fin (Linard, 2003, p. 2).

\subsection{Nouveau paradigme et nouvelle finalité de la formation}

Linard (2002) observe qu'à l'ère numérique s'entremêlent deux logiques ; la première guidée par la raison des fins, et deuxième guidée par la raison des moyens. Ainsi, la capacité d'utiliser les technologies de manière efficace devient une condition (un moyen) pour la réalisation d'apprentissages autonomes (une fin). Par ailleurs, l'autonomie serait une condition (un moyen) pour un usage efficace des technologies (une fin). Tel qu'illustré à la figure 1, le développement de l'autonomie s'incarnerait alors dans une interdépendance forte entre autonomie et usage efficace des technologies, dans un processus circulaire où basculent logique des fins et logique des moyens.

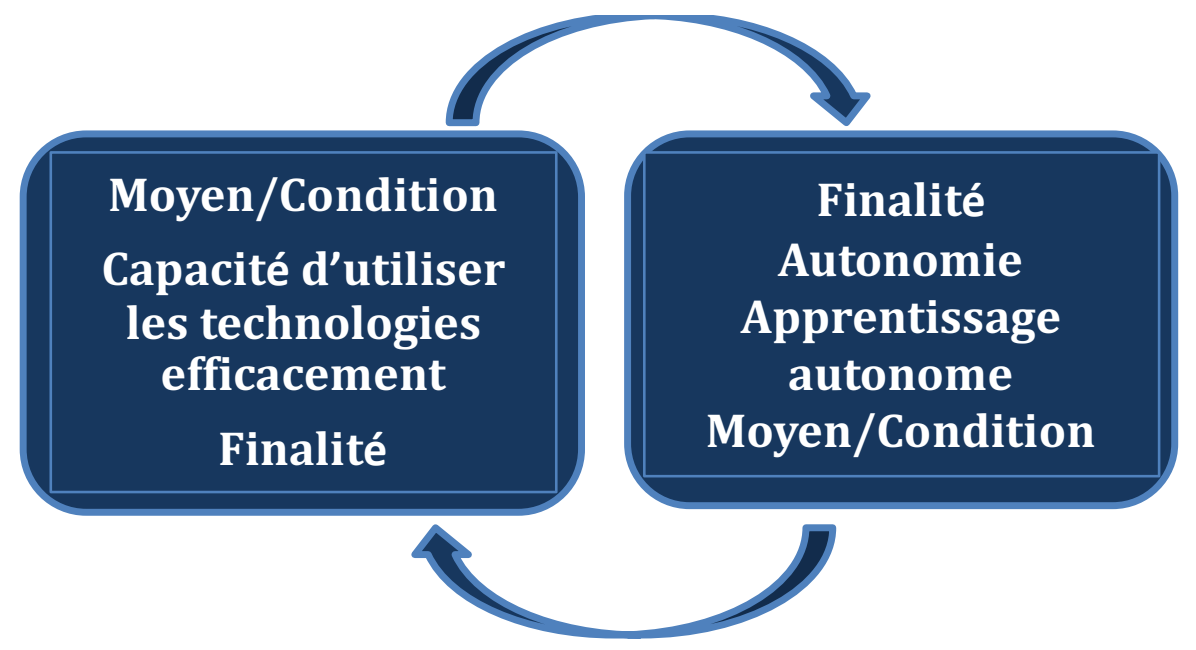

Figure 1. L'autonomie, un processus circulaire où basculent logique des fins et logique des moyens

Cette manière de raisonner les fondamentaux de la formation donne lieu à l'émergence d'un nouveau paradigme selon lequel il faut viser de manière concomitante le développement de l'autonomie et la capacité d'utiliser les technologies de manière efficace. Cette logique se distingue dramatiquement de celle voulant que la formation doive d'abord viser la maîtrise de compétences disciplinaires ou professionnelles acquises en exécutant des tâches pédagogiques tracées à l'avance dans un scénario d'apprentissage. L'apprenant s'affirme plutôt comme acteur capable de définir son projet et d'interpréter 
différents rôles au cours de son apprentissage. II se donne comme finalité d'atteindre l'autonomie, ce qui le rend apte à apprendre tout au long de la vie et se prépare ainsi à contribuer à la société.

Les changements induits par le numérique témoignent d'une nouvelle réalité tant en milieu de travail, en milieu éducatif que dans la société en général. Être autonome, clé de réussite et d'inclusion, se manifeste dans l'agir et dans la capacité d'apprendre en tout temps et en toutes circonstances. L'autonomie, concept transversal et fondateur, s'avère une nécessité, une capacité incontournable. Plus encore, l'autonomie devient une métacompétence clé pour se développer comme personne, pour se préparer à devenir des apprenants permanents, conscients et volontaires.

La capacité d'utiliser les technologies de manière efficace ne va pas de soi. Elle repose sur la translittératie, à savoir un ensemble de compétences qui vont au-delà de littératies numériques (figure 2). Thomas et ses collègues (2007) définissent la translittératie comme l'habileté à lire, écrire et interagir par le biais d'une variété de plateformes, d'outils et de moyens de communication, de l'iconographie à l'oralité en passant par l'écriture manuscrite, l'édition, la télévision, la radio, et le cinéma jusqu'aux réseaux (traduction libre). Pour Serres (2012), la translittératie est un métissage des littératies, une imbrication des cultures ; « c'est le chaudron du numérique qui brasse, mélange et remixe toutes sortes de pratiques et de cultures ». Elle est associée aux pratiques sociales, à la nature transversale des compétences induites par le numérique (compétences techniques, collaboratives, cognitives, sociales, organisationnelles ...).

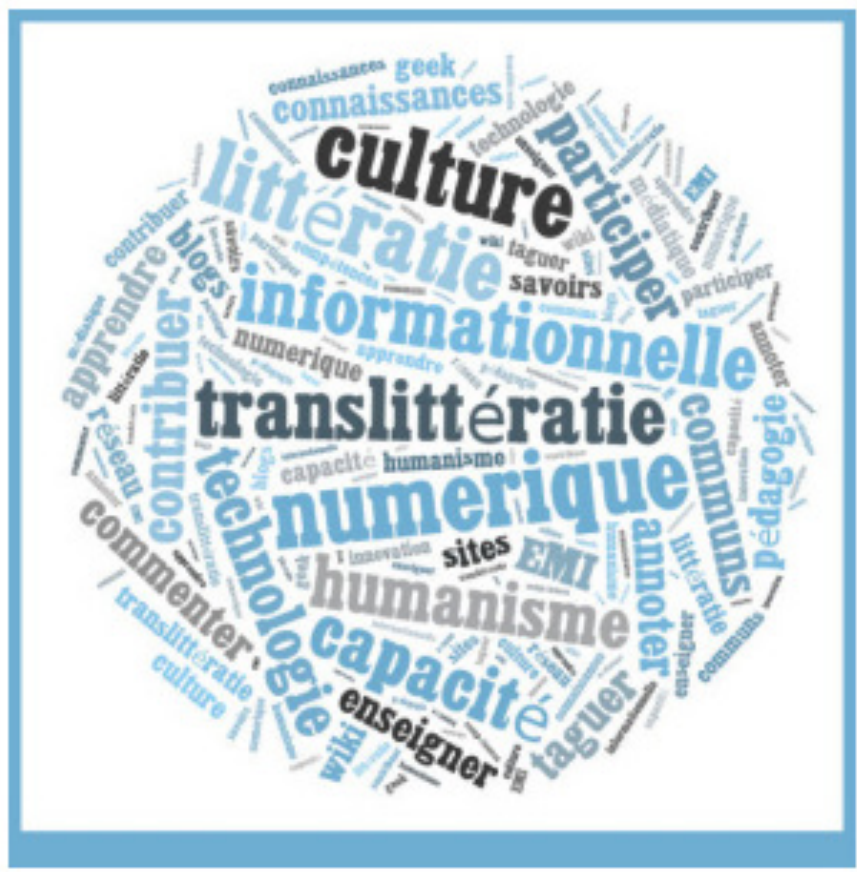

Figure 2. Translittératie

Source : Bros (2018)

Nous sommes face à un changement fondamental, voire radical, qui touche profondément les valeurs et les structures et qui suppose une transformation des conceptions de la formation et de l'apprendre. Si l'apprenant peut être considéré comme le meilleur pilote de son apprentissage évoluant dans des 
environnements qu'il aurait lui-même organisés, une réingénierie des systèmes de formation s'impose de même qu'une redéfinition fatale du rôle des divers intervenants, entre autres, celui de l'enseignant qui, comme le propose et l'illustre Duriez (2019), crée une dépendance qui n'existait pourtant pas pour l'apprentissage de la marche, de la parole ou de la manipulation des objets courants. Jacotot puis Rancière nous le rappellent : nos apprentissages les plus efficaces se sont faits sans maître! (figure 3)

De manière réaliste, ce changement de paradigme de formation est-il applicable? Est-il socialement acceptable? Est-il condamné à la marginalité? Comment pourrait-il être abordé?

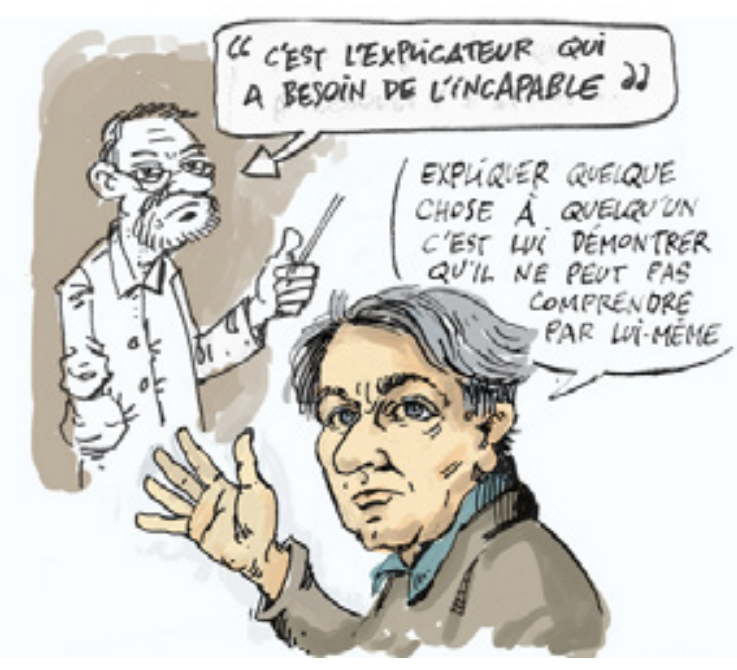

Figure 3. Je n'y connais rien, et c'est pour ça que je peux vous former... Le maître ignorant - retour sur un classique.

Source : Duriez (2019)

\section{Une ingénierie pédagogique renouvelée au service du changement}

Monique Linard (2002) proposait de repenser «l'ingénierie pédagogique qui, au nom de l'efficacité, considère les apprenants comme des agents rationnels, exécutants de tâches bien spécifiées, non pas comme des acteurs, interprètes intentionnels de rôles à géométrie variable » (p. 3). Elle envisageait une ingénierie pédagogique renouvelée qui allait supplanter les « dispositifs objectifs, impersonnels, extérieurs aux sujets, à des dispositifs subjectifs, conscients et volontaires [par des] dispositifs ouverts remaniables et détournables vers des usages imprévus » (p. 3). Cette proposition peut trouver sa place et s'insérer dans un modèle d'ingénierie plus large.

Leclercq (2003) situe l'ingénierie pédagogique dans un modèle d'ingénierie à trois niveaux. À l'échelle macrosociale, l'ingénierie sociale traduit des visées politiques et économiques sous formes de prescriptions, d'orientations, de lois, dans une visée d'intégration, d'adaptation, de changement, de reproduction sociale. Au méso-niveau, l'ingénierie de formation vise l'offre et la mise en œuvre de ce qui est promu par l'ingénierie sociale. À l'échelle microsociale, l'ingénierie pédagogique conçoit et réalise des environnements d'apprentissage à partir des prescriptions (programmes, curriculum) émanant de 
l'ingénierie de formation. Elle se rapporte à la relation pédagogique, c'est-à-dire aux processus enseigner et former qui concernent les enseignants, et au processus apprendre qui vise les apprenants. Dans cette modélisation descendante, les acteurs des niveaux méso et micro travaillent sur la base d'interprétations des prescriptions formulées au niveau qui leur est immédiatement supérieur. Ils disposent en cela d'un certain degré de liberté. Leclerc (2003) souligne en particulier la latitude des acteurs du niveau micro dont l'activité pédagogique peut être plus ou moins associée, disponible, résistante, motivée, abusée, éclairée, asservie, rebelle (§ 10) par rapport à la prescription du niveau méso.

Ainsi, loin d'être asservie à une logique descendante, l'ingénierie pédagogique pourrait devenir un puissant vecteur de changement dans les systèmes de formation en favorisant et facilitant des pratiques innovantes que les enseignants du niveau micro pourraient adopter pour ensuite les faire remonter vers les acteurs du niveau méso qui, à leur tour, pourraient les relayer à ceux du niveau macro. L'ingénierie pédagogique se positionnerait ainsi comme une force ascendante dans un modèle d'ingénierie où les frontières entre les trois niveaux sont levées, où les initiatives de l'échelon du bas sont répercutées et négociées avec les acteurs des niveaux supérieurs. Le changement ne serait pas le résultat d'une prescription, mais plutôt l'aboutissement d'une construction collective discutée et négociée par les acteurs de tous les niveaux.

\subsection{Force et faiblesse de l'ingénierie pédagogique}

L'ingénierie pédagogique a pris forme avec la montée des environnements techno-pédagogiques qui, aujourd'hui, sont d'usage courant en formation en présence ou à distance. Soucieuse d'insuffler une forme de scientificité dans la conception, elle se distancie de l'approche artisanale de conception qui ne dispose pas d'outils et de méthodes capables de traiter la complexité des environnements technologiques. L'ingénierie pédagogique se définit ainsi comme un domaine à la croisée de la conception pédagogique (design), de l'ingénierie cognitive et de l'ingénierie des systèmes d'information (Paquette, 2002). Elle en applique les principes fondamentaux qui sont ceux de la cohérence et de la systématicité. Elle emprunte à l'ingénierie cognitive ses techniques de modélisation pour définir et représenter les environnements et utilise les méthodes de l'ingénierie des systèmes d'information, garantes de la robustesse de l'environnement technologique. L'ingénierie pédagogique traite donc de manière rigoureuse la conception, le développement et la diffusion d'environnements d'apprentissage basés sur les technologies. Garante d'efficacité et de fiabilité, elle organise et structure les diverses composantes en interaction dans les environnements et formule des prescriptions relatives à l'appropriation de connaissances et à l'acquisition de compétences. Ces prescriptions se traduisent dans une suite de tâches et composent le scénario pédagogique, pièce majeure de l'environnement. L'évaluation occupe une place centrale dans la démarche de conception. Elle permet de vérifier le degré de correspondance entre la prescription pédagogique et les acquis de l'apprenant, ainsi que de mesurer de fiabilité et l'efficacité de l'environnement. Cette finalité de l'évaluation a comme avantage d'assurer que chaque apprenant pourra satisfaire les exigences de la formation dans un environnement de qualité. En revanche, l'ingénierie présente une faiblesse importante qui est celle de véhiculer une vision normalisée et transmissive de l'apprentissage, une vision qui ne laisse que peu ou pas d'espace à l'exercice de l'autonomie de l'apprenant. Dans une perspective d'autonomisation de l'apprenant, ce qui était une force s'avère une faiblesse. Comme l'écrit Linard (2002), l'approche dominante de l'ingénierie pédagogique considère les apprenants comme « des agents rationnels, exécutants de tâches bien spécifiées plutôt que comme des acteurs, interprètes intentionnels de rôles à géométrie variable » (p.3).

\subsection{Revoir les méthodes d'ingénierie pédagogique}

II existe un besoin pour des méthodes d'ingénierie pédagogique qui soient garantes de rigueur et de scientificité. II existe également le besoin de méthodes dont l'objet de conception ne se limite plus au 
contenu de connaissances et aux compétences qui s'y rapportent. II existe une demande pour des méthodes qui s'inspirent d'une logique de conception autre que celle du « cours enseigné ». On objectera que les méthodes actuelles permettent au concepteur de prendre en charge le développement de l'autonomie et l'usage efficace des technologies à l'étape du choix des stratégies pédagogiques. À cela, on peut répondre que les choix pédagogiques des concepteurs ne sont pas soumis au même traitement systématique que celui qui s'applique à la conception du contenu de formation et à son évaluation. À l'étape de l'analyse, pierre angulaire de la démarche d'ingénierie pédagogique, les besoins en matière de développement de l'autonomie et d'usage efficace des technologies ne sont pas relevés ; ils ne constituent pas des objets d'apprentissage au même titre que le sont les connaissances et de compétences disciplinaires ou professionnelles définies par les programmes de formation. Les besoins en matière d'autonomie et d'usage efficace des technologies ne sont pas considérés comme des objets formels de conception. Dans le meilleur des cas, ils font partie des préoccupations implicites du concepteur et la conception qui éventuellement s'y rapporte n'est ni documentée, ni validée par la méthode d'ingénierie.

L'ingénierie pédagogique repensée en fonction du développement de l'autonomie et d'un usage efficace des technologies, ouvertement et explicitement orientée vers un nouveau paradigme de formation répondrait à un besoin individuel, mais aussi sociétal pour la formation de citoyens autonomes capables de s'adapter au contexte économique, social et technologique qui ne cessera d'évoluer. Les environnements d'apprentissage conçus selon cette orientation « autonomisante » offriraient à l'apprenant la liberté de faire des choix, la possibilité de concevoir et de réaliser son propre projet d'apprentissage et de pratiquer l'autodirection en exerçant un contrôle sur son processus d'apprentissage (Carré, 2003, 2010; Jézégou, 2008). Dans le même esprit, ces environnements instrumenteraient le méta-apprentissage, cette capacité à mesurer, se questionner et réfléchir sur ses apprentissages (Dubois et al., 2012).

\section{En guise de conclusion. Une belle opportunité de transformation}

Le changement visant à faire des apprenants des acteurs, interprètes intentionnels de rôles à géométrie variable représente un défi important. L'apprenant aura à prendre en charge en bonne partie la responsabilité de son apprentissage. Le concepteur devra accepter l'incertitude inhérente à une pédagogie ouverte et à la liberté de choix. Les acteurs institutionnels et les décideurs politiques devront surmonter les tendances conservatrices reconnues des milieux éducatifs et de formation.

Toute proposition de changement suscite résistances et attitudes défensives aboutissant le plus souvent à des changements superficiels qui, au mieux, ont des succès de courte durée. Pour contourner cet écueil, il faudrait arriver à penser le changement autrement pour l'inscrire dans la durée et mobiliser les acteurs dans une vision fédératrice. Le changement ne s'impose ni ne se commande. II ne se résume pas à un plan. Il se conçoit d'abord comme un parcours, un cheminement dans le temps. Les problèmes et tensions qu'il suscite peuvent être exploités comme une opportunité de transformation, de croissance et de développement. Comme l'écrivait Linard (2002), il s'agit de négocier une certaine tension entre le pôle objectif, plus ou moins coercitif, de la normalisation des buts et des moyens et le pôle subjectif, plus ou moins toléré, de l'action des agents qui les mettent en œuvre (p. 3). 


\section{Liste de références}

Bros, F. (2018, 30 mars). Ce que le numérique fait à la littératie : présentation et mise en discussion de l'article " Devenir e-lettré : Quels leviers et voies d'accès à l'écrit à l'heure de la littératie numérique ? » Communication présentée au Séminaire de recherche organisé par le laboratoire méditerranéen de sociologie (LAMES, CNRS, Aix-Marseille Univ), Axe 3 "Éducation et cultures à l'ère numérique », Maison méditerranéenne des sciences de l'homme, Aix-Marseille Université. Repéré à http://www.lames.cnrs.fr/spip.php?article935

Carré, P. (2003). L'autoformation accompagnée en APP ou les sept piliers revisités. P. Carré et M. Tétart (dir.). Les ateliers de pédagogie personnalisés ou l'autoformation accompagnée en actes. Paris: L'Harmattan, 125-146.

Carré, P. (2010). L'autodirection des apprentissages. Perspectives psycho-pédagogiques. Dans D. Poisson, A. Moisan et P. Carré (dir). L'autoformation. Perspectives de recherche. (p. 117-169). Paris: PUF.

Carricano, M. \& de Lassence, G. (2009). Un usage du Text Mining : donner du sens à la connaissance client. Systèmes d'information \& management, 14(2), 85-100. https://doi.org/10.3917/sim.092.0085

Cristol, D. (2019, février 25). Bouleversements pédagogiques. Les contenus, les processus et le sens des apprentissages en mouvement. Repéré le 9 mars 2019 sur le site de Thot Cursus : https://cursus.edu/articles/42452/bouleversements-pedagogiques\#.XH1-W6BCcdV

Dubois, J., Labranche, A.-A., \& Gagné, J. (2012, décembre). Apprentissage en réseau. Consulté 14 août 2016, à l'adresse http://edutechwiki.unige.ch/fr/Apprentissage en r\%C3\%A9seau

Duriez, F. (2019, février 5). Je n'y connais rien, et c'est pour ça que je peux vous former... Le maître ignorant retour sur un classique. Repéré sur le site de Thot Cursus : https://cursus.edu/articles/42615/je-nyconnais-rien-et-cest-pour-ca-que-je-peux-vous-former\#.XI10TaBCcdU

Jézégou, A. (2008). Formations ouvertes et autodirection de l'apprenant. Savoirs, 1(16), 97-115.

Leclercq, G. (2003). Quelques usages de l'activité d'ingénierie de formation. Savoirs, 2(2), 71-104.

Linard, M. (2002). Conception de dispositifs et changement de paradigme en formation. Éducation permanente, 2002(152), 143-155.

Linard, M. (2003). Autoformation, éthique et technologies: enjeux et paradoxes de l'autonomie. Dans B. Albero (dir.), Autoformation et enseignement supérieur (p. 241-263). Repéré à l'adresse https://edutice.archives-ouvertes.fr/edutice-00000276/

Paquette, G. (2002). Modélisation des connaissances et des compétences. Québec, Qc: Presses de I'Université du Québec.

Serres, A. (2012). Repères sur la translittératie. 5ème séminaire du Groupe de Recherche sur la Culture et la Didactique de l'Information. Rennes, 7 septembre 2012.

Thomas, S., Joseph, C., Laccetti, J., Mason, B., Mills, S., Perril, S., \& Pullinger, K. (2007). Transliteracy: Crossing divides. First Monday, 12(12). Repéré à l'adresse

http://firstmonday.org/ojs/index.php/fm/article/view/206 